\title{
Giómeros fluidos en la eliminación de sensibilidad en molar permanente afectada con Hipomineralización Incisivo Molar (HIM). Reporte de caso.
}

\section{Fluid giomers in the elimination of sensitivity in permanent molar affected by Molar Incisor Hypomineralization (HIM). Case report.}

\author{
Mariella Arce-Izaguirre ${ }^{1 *}$, Gilmer Torres-Ramos ${ }^{2,3}$, María Alvino-Vales², Marya Barzola-Loayza ${ }^{2}$
}

\section{Práctica privada, Lima, Perú}

2. Área de Odontopediatría. Departamento de Odontología. Facultad de Odontología. Universidad Privada San Juan Bautista. Lima, Perú. 3. Departamento de Odontología. Facultad de Odontología. Universidad Privada San Juan Bautista. Lima, Perú.

* Correspondencia Autor: Mariella Arce Izaguirre | E-mail: mariella.arce26@gmail.com | Teléfono: +519 94978722 | Dirección: Calle Castilla la Vieja 239, Urbanización la Castellana. Santiago de Surco, Lima, Perú

Trabajo recibido el 22/01/2020.

Aprobado para su publicación el 04/04/2020

\begin{abstract}
RESUMEN
El principal motivo de consulta de los pacientes afectados con hipomineralización incisivo molar grado B $3 \mathrm{II}$, según la Academia Europea de Odontología Pediátrica (EAPO) es la estética y sensibilidad, provocando dolor a los cambios térmicos y a la hora de realizar el cepillado dental, esto puede conllevar a una rápida progresión de caries dental, por disminuir el cepillado.

En el presente caso se describe la reconstrucción y sellado de fosas profundas de la pieza 46 afectada con hipomineralización incisivo molar, mejorando la calidad de vida relacionada a la salud bucal del paciente y eliminando la sensibilidad en una sola cita. PALABRAS CLAVE:
\end{abstract}

Defecto de esmalte; Desmineralización dental; Sensibilidad de la dentina; Hipoplasia de esmalte dental.

Int. J. Inter. Dent Vol. 13(2); 95-98, 2020.

\section{ABSTRACT}

The main reason for consultation of patients affected by molar incisor hypomineralization level B 3 II (EAPO) is aesthetics and sensitivity, causing pain with thermal changes, when consuming cold drinks and when brushing the teeth. This can lead to a rapid progression of tooth decay, by decreasing the brushing.

In the present case, the reconstruction and sealing of deep pits of the tooth 46 affected by molar incisor hypomineralization are described, improving the quality of life related to the patient's oral health and eliminating sensitivity in a single appointment.

KEYWORDS:

Enamel defect; Dental demineralization; Dentine sensitivity; Dental enamel hypopasia.

Int. J. Inter. Dent Vol. 13(2); 95-98, 2020.

\section{INTRODUCCIÓN}

La hipomineralización de incisivos molares $(\mathrm{MIH})$ es un defecto del desarrollo, un proceso de mineralización disfuncional del esmalte que afecta de uno a cuatro primeros molares permanentes, además, los incisivos permanentes superiores e inferiores también pueden verse afectados ${ }^{(1)}$.

$\mathrm{Rao}^{(2)}$ tiene una etiología multifactorial (factores ambientales, médicos, genéticos, sistémicos), todos los elementos etiológicos actúan adicionalmente o incluso sinérgicamente con predisposición genética, y estos pueden estar relacionados con los períodos prenatales, perinatales y post natales del desarrollo infantil.

Melénde $z^{(3)}$ clínicamente, la hipomineralización se caracteriza por opacidades de diversos tamaños y decoloración de blanco a amarillo/ marrón, van a tener un espesor de esmalte normal y una demarcación definida entre el esmalte afectado y el esmalte sano, y estas son más frecuentes en las superficies oclusal y bucal.

Ghanim $^{(4)}$ para realizar un buen diagnóstico, debemos tener en cuenta la codificación de la hipomineralización Incisivo Molar (EAPO) esta codificación se divide en tres criterios de evaluación, va a evaluar el estado de erupción del diente, dándole la codificación de "A" a una pieza dental no visible o con menos de un tercio de erupción de la superficie incisal u oclusal, según sea el caso, la codificación "B" será para una pieza con erupción completa o con más de un tercio de superficie. El segundo criterio a evaluar serán los criterios clínicos, dentro de los cuales tenemos la siguiente codificación: "0" para piezas dentales sin defecto del esmalte; "1" para piezas dentales con defecto del esmalte pero que no son HIM ("11" opacidad difusa como fluorosis, "12" hipoplasia, "13" amelogénesis imperfecta, "14" defecto de hipomineralización no HIM como diente de Turner); "2" para piezas con opacidades demarcadas ("21" blanco o crema, "22" amarillo o marrón); "3" cuando se presentan fracturas o pérdida de estructura; "4" cuando la pieza ha sido restaurada y encontramos una restauración atípica; " 5 " hay presencia de caries atípicas; "6" cuando la pieza fue extraída por lo agresivo de la HIM y no se pudo dar tratamiento; "7" no categorizado. Y el último criterio a evaluar es la extensión de la lesión, la que será codificada con números romanos de la siguiente manera, "I" cuando está afectada menos de un tercio de la pieza dental; "II" cuando está afectada un tercio o más, pero menos de dos tercios de la pieza dental; "III" cuando la pieza dental tiene más de dos tercios de HIM.

Ghanim $^{(4)}$ dentro de las opciones de tratamiento en piezas diagnosticadas con HIM "B 3 II", tenemos las restauraciones con ionómero de vidrio, restauraciones con giómeros y restauraciones con resina, si la extensión hubiese sido de más de 2 tercios de la pieza dental se habría podido optar por coronas de acero.

Shofu(5) el termino Giómeros (S-PRG) proviene de Glass lonomer 
(lonómero vítreo) + Composite. Los Giómeros fluidos, son una tecnología relativamente nueva, que emplea el ionómero de vidrio previamente reaccionado y resina compuesta, dentro de su composición, lo que permite obtener las propiedades de ambos: del ionómero de vidrio, la liberación y recarga de flúor y de la resina, la estética, la fácil manipulación y sus propiedades físicas. Dentro de sus ventajas tenemos: recarga y liberación de flúor, estética parecida a los dientes naturales, efecto antiplaca, alta radiopacidad, fluorescencia, estabilidad clínica, resistencia al desgaste en dientes posteriores.

\section{REPORTE DE CASO CLÍNICO}

Paciente de género masculino de 07 años, 10 meses de edad, llegó a consulta con su mamá al Policlínico Dental de la Universidad Privada San Juan Bautista de Lima-Perú; refiriendo que el niño, presenta mucha sensibilidad, dolor al momento de ingerir sus alimentos y al realizar la higiene dental; le incomoda que el diente se vea de color amarillomarrón. Al examen clínico extraoral (figura 01) no presenta ninguna alteración. Al evaluar el comportamiento (Escala de Frankl) el niño presentó comportamiento positivo. Al examen clínico intraoral (figura 02) el paciente presenta dentición mixta primera fase, erupción parcia de las primeras molares permanentes. varias piezas con fosas y fisuras profundas y también con HIM (B 3 II), la pieza 46 presenta fractura dental en superficie oclusal y vestibular, se observa $2 / 3$ de erupción, que se diagnostica con HIM grado B 3 II (EAPO) debido a la distribución y forma de las manchas amarillo-marrón que presenta la pieza dental afectada. El pronóstico es reservado, a pesar de la colaboración del paciente y la persistencia de la mamá, ya que la pieza dental seguirá el curso de la enfermedad fracturándose, hasta que termine el proceso de erupción. No se requiere exámenes auxiliares. Se determinó realizar el tratamiento con Giómeros fluidos, para reconstruir la pieza, sellar fosas profundas y eliminar la sensibilidad en una sola sesión, por los beneficios antes mencionados.

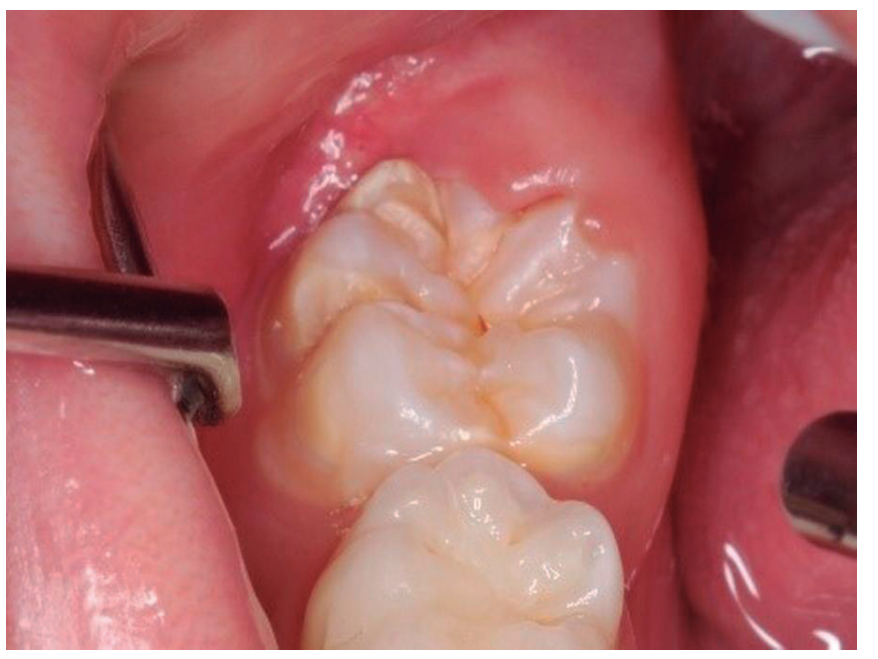

Figura 1. Pieza 46 con HIM (B 3 II) (B: Erupcionado, 3: Perdida de estructura, II: $1 / 3$ a $2 / 3$ de extensión), con fisuras y fosas profundas.

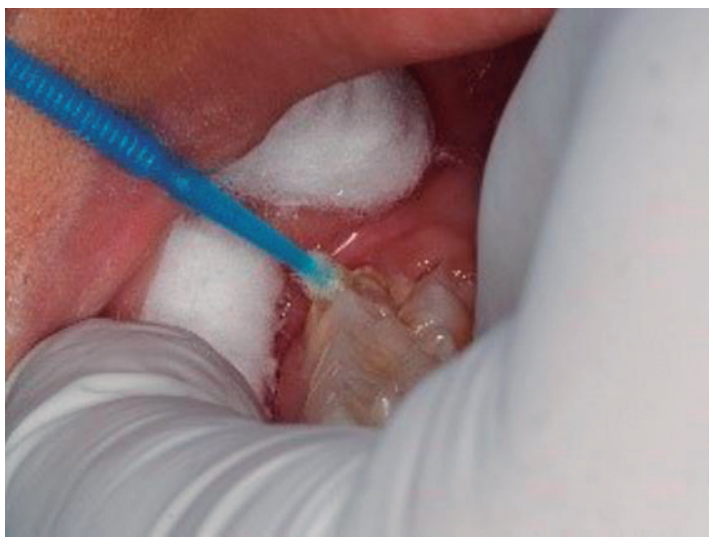

$\overline{\text { Figura 2. Aislamiento relativo, aplicación del Brix } 3000 \text { por } 30 \text { segundos y }}$ remoción con cureta (Mayleffer $\mathrm{N}^{\circ} 46$ ).
Luego de considerar las medidas de bioseguridad se procedió con el tratamiento de la pieza 46 (Figura 1), se realiza aislamiento relativo por la falta de erupción total de la pieza y la excesiva sensibilidad del paciente, aplicó brix 3000 (gel enzimático para la remoción a traumática de tejido infectado) (brix SRL Argentina), sobre las superficies infectadas de la pieza 46, luego de 2 minutos, con una cureta de dentina (Mayleffer $\mathrm{N}^{\circ}$ 46) eliminamos estructura dental con HIM (Figura 2), se lavó con agua, y secó, se realizó desproteinización de la cavidad dentaria con una bolita de algodón embebida con hipoclorito de sodio al $5 \%$ por 30 segundos, y se enjuagó con una bolita de algodón embebida en agua, secado con bolitas de algodón (Figura 3), luego de cambiar el aislamiento relativo; se aplicó el adhesivo de 7ma generación Palfique Bond (Tokuyama), por la sensibilidad del paciente, se airea, fotocura por 20 segundos (Figura 4), y se aplica Giómero fluido F00 de reconstrucción (Beautifil Flow Plus de Shofu inc.), dando forma con el bruñidor para resina cabeza de huevo y se fotocuró por 20 segundos (Figura 5). Se utilizó el Giómero fluido F03 de sellado de fosas y fisuras (Beautifil Flow Plus de Shofu inc.) fotocurando por 20 segundos (Figura 6). Finalmente se comprueba el ajuste oclusal con una fresa cilíndrica plana (108-009) eliminando excesos y porosidades (Figura 7a).

Se realizó el control de la pieza dental a los 7 meses observándose en perfectas condiciones anatómicas y funcionales. (Figura 7b)

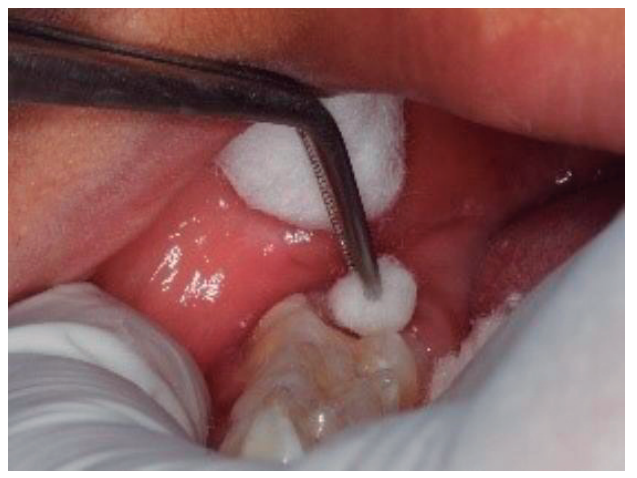

Figura 3. Desproteinización con hipoclorito de sodio al $5 \%$ por 30 segundos, lavado y secado con bolitas de algodón.



Figura 4. Aplicación de adhesivo Palfique $7^{\circ}$ generación, aireado y fotocurado por 20 segundos.



Figura 5. Reconstrucción cara ocluso vestibular con Giómero F00, y modelado con bruñidor cabeza de huevo y fotocurado por 20 segundos. 


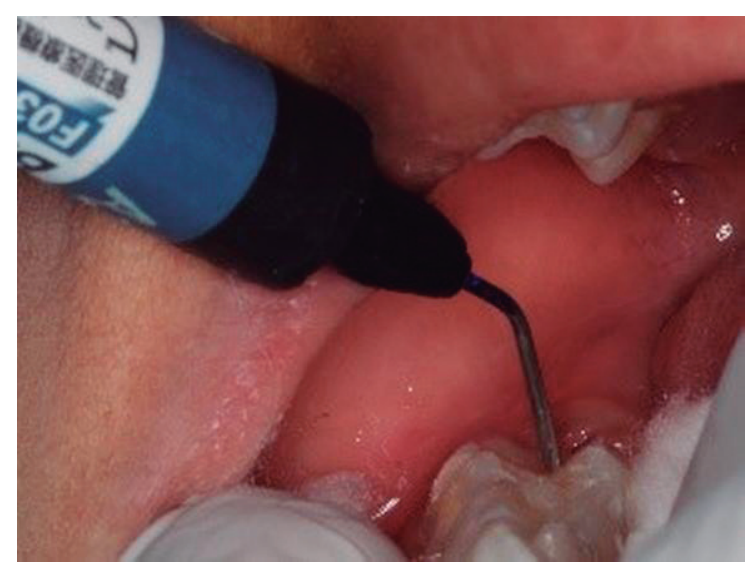

Figura 6. Sellado de fosas y fisuras con Giómero 03, fotocurado por 20 segundos.

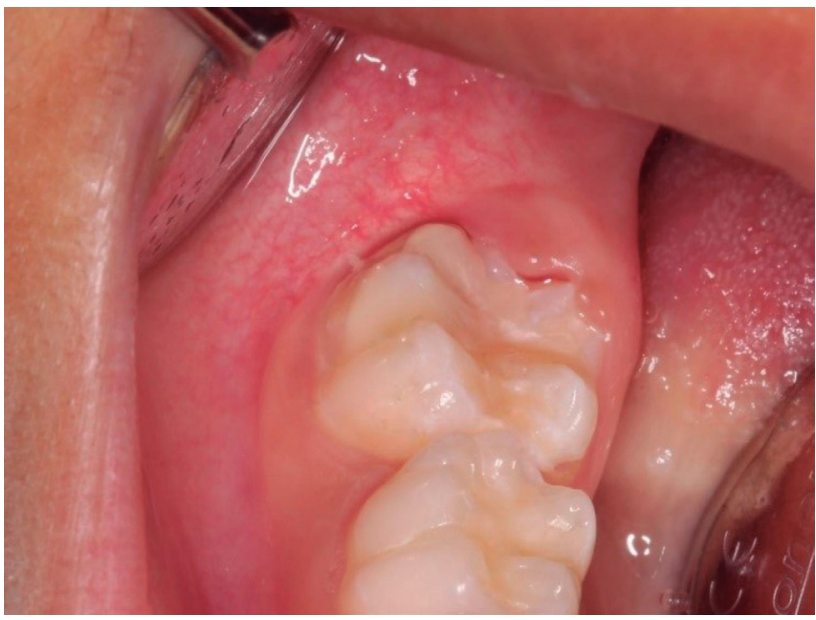

Figura 7a. Control de oclusión y anatomía dental.

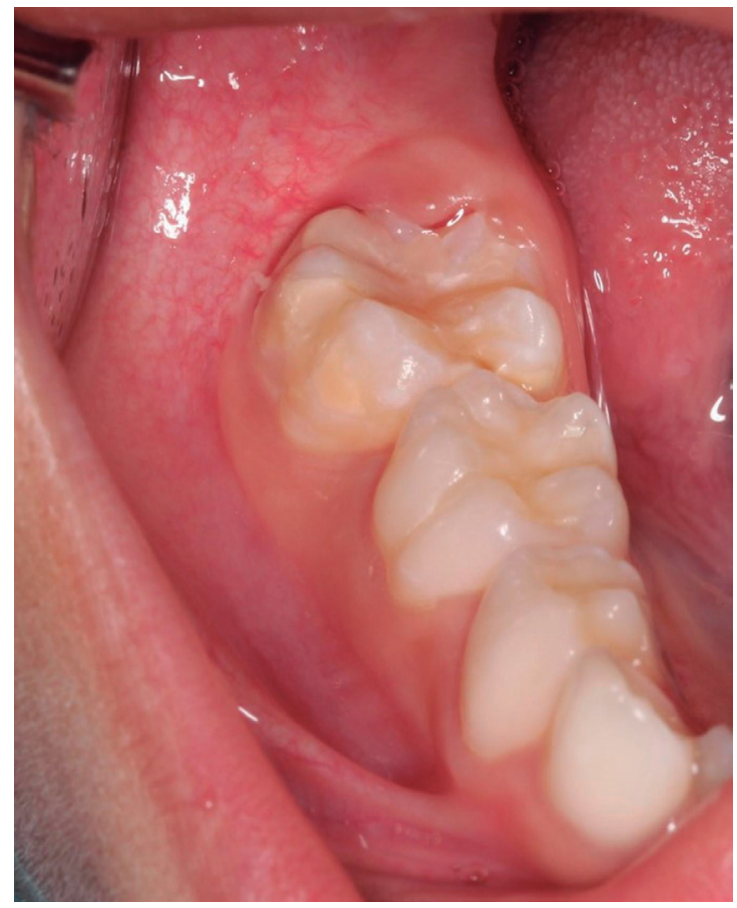

Figura 7b. Control a los 7 meses.

\section{DISCUSIÓN}

Jauregui(6) en el 2017 usó giómeros fluidos para el sellado de fosas y fisuras, usándolo como un sellante convencional en molares sanos, observó que los sellantes colocados a pacientes a base de giómeros fluidos, se mantienen adaptados con buena estabilidad en el color, efecto antiplaca y un buen control de caries. En este reporte de caso usé giómeros fluidos F03 para sellar una molar permanente con HIM, por las propiedades antes mencionadas.

Suárez-Carranza(7) en el 2016 usó giómeros fluidos y densos para la reconstrucción en piezas con HIM en el sector anterior, por ser una técnica eficaz, segura y conservadora para enmascarar este tipo de lesiones, preservando al máximo la estructura dentaria, además por su propiedad del material de absorber y liberar bajas concentraciones de flúor, nos asegura una restauración en constante proceso remineralizante, evitando el acumulo de placa bacteriana dándole una mejor expectativa al paciente. Es por eso que en este reporte de caso usé giómeros fluido F00 para la reconstrucción de una pieza en el sector posterior con HIM.

Guillet y cols. ${ }^{(8)}$ en el 2002, realizaron un estudio in vitro para comparar una resina fluida (Tetric Flow), con un sellante convencional (Helioseal F), observando que el primero fue más eficiente al compararlo con el segundo, logrando obturar mejor las fosas y fisuras de los dientes. El inconveniente con las resinas fluidas es que no liberan flúor en comparación con otros selladores convencionales. En este reporte de caso usé giómeros fluidos por las propiedades que tiene como la estética de la resina y la liberación de flúor de los ionómeros, que sería lo más adecuado para sellar las fosas profundas.

Dhull(9) en el 2009, en la India, comparó la cantidad de liberación de fluoruro de giómeros y compómeros, utilizando diferentes regímenes de fluoruros tópicos, obteniendo como resultado que la mayor liberación de flúor (ppm) se encontró en giómeros cuando se compara con compómeros, además se encontró que el aumento de la exposición al fluoruro aumentó significativamente la liberación de flúor de los giómeros y compómeros. Es por eso que en este reporte de caso se usaron giómeros para la reconstrucción de superficies infectadas.

Abdul $^{(10)}$ en el 2012 comparó diferentes ionómeros de vidrio y materiales restauradores con la compresión, liberación y recarga de flúor de los giómeros, siendo estos últimos los que liberaban mayor cantidad de flúor después de los ionómeros de vidrio. Es por eso que en este reporte de caso usé giómeros, por las propiedades estéticas de las resinas y la gran liberación de flúor ya que se trata de una pieza con HIM.

Hotwani(11) en el 2014 comparó la estabilidad de color de las restauraciones, al someterlos a la inmersión en bebidas de la preferencia de niños, comparando piezas con restauración de ionómero de vidrio (Fuji II) y giómero (Beautil II), siendo el giómero el que mostró mayor resistencia a las manchas, en periodos de tiempo absoluto. Es por eso que en este reporte de caso se usaron los giómeros, ya que al paciente le molestaba la apariencia de la pieza dental, pudiendo mantener la obturación sin cambios de color y liberando flúor progresivamente.

de Oliveira ${ }^{(12)}$ en el 2015 , usó ionómero de vidrio, para tratar una molar permanente con HIM, por la liberación de flúor, posteriormente esta curación fue reemplazada por resina, debido al desgaste, la filtración y la caries recurrente. En este reporte de caso utilicé giómero fluido F00 para la reconstrucción, por las propiedades como antiplaca, liberación de flúor, y resistencia al desgaste.

Azarpazhooh ${ }^{(13)}$ en el 2008 usó para los pacientes con HIM aplicación de barniz fluorado, esta modalidad tiene gran importancia en la etapa temprana post-eruptiva, cuando el diente defectuoso es más vulnerable a la ruptura y al ataque de caries. En la última etapa post-eruptiva, si todavía está intacto, el diente debería haber madurado y sólo debería necesitar control periódico y aplicación de barniz fluorado cada 6 meses. En este reporte de caso no se pudo usar esa alternativa, ya que la pieza presentaba fractura y pérdida de tejido.

Sundfeld ${ }^{(14)}$ en el 2007 determinó que los defectos de color amarillo o marrón-amarillo son de espesor total, mientras que los de color amarillo cremoso o blanquecino-crema son menos porosos y de profundidad variable. Para su tratamiento utilizó blanqueamiento con peróxido de carbamida y microabrasión con ácido clorhídrico al $18 \%$ o fosfórico al $37,5 \%$. Sin embargo, en pacientes menores de 18 años puede inducir hipersensibilidad, irritación de la mucosa y pérdida del esmalte. En este reporte de caso no se pudo usar este tratamiento por la edad del paciente y por la excesiva sensibilidad que presentaba.

William ${ }^{(15)}$ en el 2006 utilizó coronas metálicas preformadas (PMC). Proporcionando una completa cobertura de molares defectuosos, evitando la pérdida de dientes, controlando la sensibilidad, no es costoso y requiere poco tiempo para la preparación dentaria y cementación, sin embargo, puede ser necesaria una reducción severa de los tejidos dentales para la inserción de las coronas, por lo que se recomienda su uso en piezas totalmente erupcionadas. En este reporte de caso no se 
pudo usar esta técnica, por que la pieza estaba en proceso de erupción.

\section{CONCLUSIÓN}

En el presente reporte se utilizó giómeros fluidos en una pieza posterior(46) diagnosticada con HIM (B 3 II) (B: Erupcionado, 3: Pérdida de estructura, II: $1 / 3$ a $2 / 3$ de extensión), con fosas y fisuras profundas, alcanzando un gran éxito, reconstruyendo los tejidos perdidos, eliminando la sensibilidad y devolviendo la función.

\section{RELEVANCIA CLÍNICA}

El uso de Giómeros en el tratamiento de piezas con HIM que están en proceso de erupción, son relevantes porque nos permiten principalmente reconstruir los tejidos perdidos y eliminar la sensibilidad dental en una sola cita; teniendo una liberación constante de flúor, siendo más económico y resistente que las resinas y los ionómeros de reconstrucción convencionales.

\section{CONFLICTO DE INTERÉS}

Certificamos no tener conflictos de interés y que el estudio fue financiado por nosotros.

\section{Bibliografía}

1. da Cunha Coelho ASE, Mata PCM, Lino CA, Macho VMP, Areias CMFGP, Norton APMAP, et al. Dental hypomineralization treatment: A systematic review. J Esthet Restor Dent. 2019;31(1):26-39.

2. Rao MH, Aluru SC, Jayam C, Bandlapalli A, Patel N. Molar incisor hypomineralization. J Contemp Dent Pract. 2016;17(7)609-13.

3. Meléndez-Trigoso M. "Prevalencia de la hipomineralización incisivo molar en niños de $6-12$ años de la Institución Educativa Manuel Fernando Bonilla y del Puericultorio Pérez Araníbar. Lima - Perú 2017" [tesis para obtener el título de cirujano dentista]. Lima, Universidad Científica del Sur, 2018.

4. Ghanim A. Silva MJ, Elfrink M, Lygidakis N, Marin R, Weerheijm K, Manton D. Molar incisor hypomineralisation (HIM) Training manual for clinical field surveys and practice. Eur Arch Paediar Dent. 2017;178:225-242.

5. Buscador de materiales odontológicos. Lima: Shofu Dental. [citado 22 abril 2019] disponible en: http://www.shofu.com/es/products/restoratives/restorative/aboutgiomer/

6. Jauregui-Paredes J. Manejo odontológico preventivo, con aplicación de giomeros fluidos. Reporte Clínico [Para optar el Título de Segunda Especialidad Profesional en Odontopediatría], Lima, Universidad Nacional Mayor De San Marcos Facultad de Odontología Unidad de Posgrado, 2017.

7. Suárez-Carranza A, Torres-Ramos G. Nuevos materiales restauradores con liberación de flúor en manejo rehabilitador de odontopediatría. Reporte de caso. Simiykita. 2016 Jul-Dic;2(2):98-104.
8. Guillet D, Dupuis V, Dorignac G. Microleakage and penetration depth of three types of materials in fissure sealant: self-etching primer vs. Etching: an in vitro study. J Clin Pediatr Dent. 2002 Winter;26(2):175-8.

9. Dhull KS, Nandial B. Comparative evaluation of fluoride release from PRGcomposites and compomer on application of topical fluoride: an in-vitro study. $J$ Indian Soc Pedod Prev Dent. 2009;27(1):27-32.

10. Abdul SM, Shamsul M, Bashar AKM, Abdu I, Al-Mansur MA. Compressive strength, fluoride release and recharge of giomer. Updat Dent Coll J. 2012;2(2):28-37 11. Hotwani $\mathrm{K}$, Thosar N, Baliga S. Comparative in vitro assessment of color stability of hybrid esthetic restorative materials against various children's beverages. J Conserv Dent. 2014;17:70-4

12. de Oliveira DC, Favretto CO, Cunha RF. Molar incisor hypomineralization: Considerations about treatment in a controlled longitudinal case. J Indian Soc Pedod Prev Dent. 2015;33:152-5.

13. Azarpazhooh A, Limeback H. Clinical efficacy of casein derivatives: a systematic review of the literature. J Am Dent Assoc. 2008;139(7):915-24.

14. Sundfeld RH, Croll TP, Briso AL, de Alexandre RS, Sundfeld Neto D. Considerations about enamel microabrasion after 18 years. Am J Dent. 2007;20(2):67-72.

15. William V, Burrow MF, Palamara JE, Messer L. Microshear bond strength of resin composite to teeth affected by molar hypomineralisation using 2 adhesive systems. Pediatr Dent. 2006;28:233-241. 